\title{
Immunomodulation of Glatiramer Acetate in Multiple Sclerosis
}

\section{Chunhua Chen*}

Department of Biochemistry and Molecular Biology, Mayo Clinic, Rochester, MN, USA

\begin{abstract}
Multiple sclerosis (MS) is a chronic inflammatory autoimmune disease in central nervous system (CNS) characterized by demyelination as well as axonal and neuronal degeneration. Glatiramer acetate is a mixture of synthetic polypeptides comprising four amino acids resembling the myelin basic protein (MBP), and approved as an immunoregulatory drug for the treatment of relapsing-remitting MS. The mechanism of action of GA in MS patients and the animal model experimental autoimmune encephalomyelitis (EAE) were extensively investigated over years. The cumulative findings indicate GA exerts its therapeutic activity by immunomodulating various levels of the immune response. This includes the blockade of major histocompatibility complex (MHC) molecules, T cell receptor antagonist, induction of GA-specific suppressor Th2 cells, an increase in frequency and function of CD4+CD25+FoxP3+ regulatory T cells, the down-regulation of Th1 and Th17 differentiation; the development of type II antigen presenting cells (APCs). In the review, we aim to provide a comprehensive overview of the immunoregulatory properties of GA in adaptive and innate immune response, in particular on the CD4+ effector T cells.
\end{abstract}

Keywords: Glatiramer acetate; Multiple sclerosis; Immune regulation; $\mathrm{T}$ cells

\section{Multiple Sclerosis}

Multiple Sclerosis (MS) is a chronic inflammatory demyelinating disease of central nervous system (CNS), characterized by myelin destruction, loss of oligodendrocytes, axonal damage and astrogliosis [1]. It is the most common neurological disorder among young adults in which women are affected twice as frequently as men [2]. The exact etiology of MS remains unknown. Much progress has been made in understanding its pathology. Current concepts assume that the pathogenesis of MS involve multiple factors including genetic predisposition, environmental factors, immune dysregulation, and viral infections. The breakdown of immune tolerance to self-antigens in genetically susceptible individuals is thought to be a key event in the development of MS $[3,4]$.

\section{Immunopathogenesis of multiple sclerosis}

The evidence from the animal model of MS, experimental autoimmune encephalomyelitis (EAE) and clinical data from MS patients support the notion that MS occurs as a consequence of the activation of autoreactive myelin-specific $\mathrm{T}$ helper (Th) cells. It is likely that exposure to an unknown microbial antigen, which contains protein sequences cross-reactive with self-myelin antigens, results in the activation of myelin-specific T cells. Activated T cells subsequently released pro-inflammatory cytokines, such as tumor necrosis factor- $\alpha$ (TNF- $\alpha$ ), interferon- $\gamma$ (IFN- $\gamma$ ) and Interleukin-1 (IL-1) in the periphery. These activated cells undergo adhesion to the endothelia barrier, attraction, and active invasion into CNS. In the CNS, myelin specific $\mathrm{T}$ cells can be further activated by local and infiltrating antigen presenting cells. Reactivation of these cells results in increased secretion of pro-inflammatory cytokines and chemokines, which in turn recruit and activate macrophages and other inflammatory cells. Activated $\mathrm{T}$ cells may directly attack the oligodendrocytes and destroy the myelin. Activated macrophages/microglia can secrete toxic molecules (e.g., nitric oxide) to further enhance myelin destruction.

\section{The Pathogenic Role of CD4 T Helper Cell Subsets in MS}

CD4+ T helper (Th) cells are essential regulators of immune responses and inflammatory diseases. Based on their cytokine secretion and transcription factor expression, $\mathrm{CD}^{+}$Th cells can be divided into several subsets: Th1, Th2 and Th17. Th1 cells secrete IFN- $\gamma$ and promote cell-mediated immunity against intracellular pathogens; Th2 cells secrete IL- 4 and IL-10 and mediate humoral immunity and defend against extracellar pathogens and parasites; Th17 cells that producing IL-17 participate in the autoimmunity and tissue inflammation, protect the host against certain pathogens.

In the past years, Th1 cells are thought originally to be the main pathogenic T cells in MS and its animal model EAE, while Th2 cells are thought to be protective. Th 1 cells secreting IFN- $\gamma$ are closely associated with the clinical severity of EAE and could independently induce EAE when adoptively transferred into naïve mice [5]. Recently, accumulating evidence indicates that a new-identified Th subset: Th17 cells play an important role in the pathogenesis of MS and EAE. Th17 cells were found in the brain lesions of patients with MS; IL-17 expression was elevated in the serum and cerebrospinal fluid (CSF) of patients with MS [6]. IL-17 knockout mice show a significant, but not complete, reduction in severity of EAE. Administration of neutralizing anti-IL-17 antibody in vivo reduced the severity of EAE [7,8]. Further, adoptive transfer of Th17 cells directly induced severe EAE in mice. The evidence supported that Th17 and Th1 cells both attributes to the pathogenesis of MS and EAE.

CD4+CD25+Foxp3+ regulatory $\mathrm{T}$ cells (Treg) are an important subclass of regulatory cells that maintain immune tolerance by suppressing self-reactive Th cells. Forkhead transcription factor Foxp3 is the key transcription factor in the physiological development of Treg [9]. There is evidence to suggest that the function of Treg cells in MS patients is impaired. Their inhibitory effect on antigen-specific

*Corresponding author: Chunhua Chen, Department of Biochemistry and Molecular Biology, Mayo Clinic, Rochester, MN, USA, E-mail: Chen.Chunhua@mayo.edu

Received April 23, 2016; Accepted May 09, 2016; Published May 12, 2016

Citation: Chen C (2016) Immunomodulation of Glatiramer Acetate in Multiple Sclerosis. Neurochem Neuropharm Open Access 1: 110.

Copyright: $\odot 2016$ Chen C. This is an open-access article distributed under the terms of the Creative Commons Attribution License, which permits unrestricted use, distribution, and reproduction in any medium, provided the original author and source are credited. 
$\mathrm{T}$ cell proliferation was significantly reduced compared to healthy individuals [10].

\section{Development of Glatiramer Acetate}

Glatiramer acetate (also known as GA, Copaxone) is a synthetic random copolymer composed of the four amino acids L-alanine, L-lysine, L-glutamate and L-tyrosine in a defined molar ratio of 4.2:3.4:1.4:1.0 with a length of 40-100 residues. It was first synthesized to mimic the encephalitogenic properties of myelin basic protein (MBP), one of the major myelin autoantigen involved in the induction of EAE, the animal model of MS. Instead of inducing EAE disease, it has been shown to be effective in preventing and suppressing EAE by various encephalitogens in a variety of species $[11,12]$. GA has also been shown to have beneficial effects on the clinical course and MRIdefined brain lesions of patients with multiple sclerosis. In 1996, GA is approved as an immunoregulatory drug by the United States Food and Drug Administration (FDA) for the treatment of relapsing-remitting MS that slows the progression of disability and reduces relapse rate [13].

\section{Mechanism of action of GA in MS}

The mechanism by which GA induces its beneficial effect in EAE animals and MS patients was largely investigated for many years. These studies demonstrate that GA has unique immune regulatory properties on adaptive and innate immune system. We compile the current knowledge on the immunomodulatory mechanism of GA in the treatment of MS and EAE.

\section{Competition with MBP for binding to MHC molecules}

It was demonstrated that GA exhibits a high-affinity and promiscuous binding to a variety of major histocompatibility complex (MHC) class II molecules on antigen presenting cells (APC). GA does not undergo the processing before binding to MHC molecules. GA may compete with myelin-basic protein (MBP) for binding to MHC II molecules on the surface of APC cells, and even displaced the MBP antigen when already bound. This competition for binding to the $\mathrm{MHC}$ can consequently lead to the inhibition of myelin-reactive $\mathrm{T}$ cell response $[14,15]$.

\section{Antagonism at the $\mathrm{T}$ cell receptor of myelin specific $\mathrm{T}$ cells}

Aharoni et al. reported that GA was shown to inhibit the response to the immunodominant epitope of MBP peptide $82-100$ by acting as a T cell receptor (TCR) antagonist. The immunodominant determinant $82-100$ of MBP, a major target of T cells in brain lesions of MS patients. In contrast to the broad specificity of the MHC blocking induced by GA, its TCR antagonistic activity was restricted to the 82-100 determinant of MBP [16].

\section{Induction of GA-specific suppressor Th2 cells}

In untreated MS patients and healthy subjects, the majority of GA-reactive CD4+ T cells belong to the Th1 subset. Th1 cells characteristically produce a spectrum of pro-inflammatory cytokines such as IFN- $\gamma$, IL-2 and IL-12 [17]. During treatment with GA, the cytokine profile of the GA-reactive T cells shifts towards the Th2 type [18]. GA-reactive Th2 cells produce anti-inflammatory cytokines, including IL-4, IL-5, IL-6 and IL-13 [19,20]. These cells migrate into the CNS, and reactivate locally by cross-reactivity with myelin antigen. After local re-stimulation, GA-reactive Th2 cells secrete antiinflammatory cytokines and inhibit the action of encephalitogenic $\mathrm{T}$ cells, which dampen the local inflammation process. The process is termed "bystander supression" [21,22]. These GA-specific Th2 cells can produce neurotrophic factors BDNF, which might favor remyelination and axon protection [23-25].

\section{Regulation of GA on CD4+ Th cells}

Th1 and Th17 are pathogenic $\mathrm{T}$ cells in the development of MS and EAE. Kantengwa et al. reported that GA inhibited Th1 differentiation of CD4+ T cells at various $\mathrm{T}$ cell maturation stages and in an antigen-independent manner [26]. In vivo GA treatment biased differentiation of CD4+ T cells from the detrimental Th1 phenotype towards the anti-inflammatory Th2 phenotype [27]. Recently, our study has demonstrated GA inhibited Th17 differentiation through down-regulation of STAT3 phosphorylation and transcription factors ROR $\gamma t$ and ROR $\alpha$ expression in vivo. In vitro human and mouse Th17 differentiation system, GA inhibited the differentiation of Th17 in a dose-dependence manner. In Th1 differentiation system, GA also suppressed Th1 differentiation in vitro. Further, we investigated which Th subset (Th17 or Th1) was chiefly responsible for the treatment effect of GA. Our data indicated that the treatment effect of GA in EAE was mainly attributable to its regulatory property on Th17 differentiation [28].

CD4+CD25+Foxp3+ regulatory $\mathrm{T}$ cells have the beneficial effect in the development of MS by suppression of pathogenic T cells. Viglietta et al. reported that the effector function and the frequency of Treg is significantly decreased in the peripheral blood of patients with MS [29]. Several studies provided the evidence that GA have the beneficial effect on induction of $\mathrm{CD} 4+\mathrm{CD} 25+$ Treg cells. In vitro human and animal system, GA induced the conversion of peripheral CD4+CD25to $\mathrm{CD} 4+\mathrm{CD} 25+$ regulatory $\mathrm{T}$ cells through the activation of transcript factor Foxp3. GA treatment led to a significant increase in Foxp3 expression in CD4+ T cells in MS patients whose Foxp3 expression was reduced at baseline [30].

\section{Regulation of GA on APC cells}

In the past, it is considered that $\mathrm{T}$ cells are primary target of GA. Early studies focused on its influence on the adaptive immune system. APCs including monocytes and dendritic cells (DCs) play the central role in the initial and development of immune response. The interaction between APCs and T cells is fundamental for any adaptive $\mathrm{T}$ cell immune response. More recent studies indicate that GA may affect the properties of APCs. Vieira et al. reported that in vitro DCs exposure to GA have an impaired capacity to secrete Th1 polarizing factor IL-12p70, therefore preferentially induce Th2 cells and enhanced levels of the anti-inflammatory cytokine IL-10 [31,32]. Further, Kim et al. reported that mococytes from GA-treated patients produced significantly higher amounts of IL-10 and lower amounts of IL-12. GA therapy leads to the generation of type II monocyte, which contributes to Th2 deviation both in the periphery and CNS of MS patients [33]. Weber et al. also reported that lipopolysaccharide (LPS)induced activation marker CD150/SLAM expression and TNF- $\alpha$ production were significantly reduced in monocytes from GA-treated patients compared with controls [34]. These studies clearly indicated that GA treatment promotes Th2 cells differentiation by modifying the phenotype of APCs. Our study has demonstrated that GA inhibited Th17 differentiation by the reduction of IL- 6 in treated monocytes. GA primarily interacts with monocytes and inhibits the production of IL-6, critically required for Th17 differentiation through STAT3 activity in T cells [28]. These findings provide the possibility that GA treatment may compromise innate immune responses in GA-treated MS patients.

\section{Conclusion}

Glatiramer acetate is a random polymer of four amino acids enriched in myelin basic protein, and approved as an immunomodulatory drug for the treatment of relapsing MS. It has unique immune regulatory 
properties on adaptive and innate immune system. GA regulates the immune response at different levels, including binding to MHC II molecules as MHC blocker and TCR antagonist; preferential Th2 deviation in CD4 T cells; the down-regulation of Th1 and Th17 differentiation; restoration of frequency and function of Treg cells; biasing dendritic cells and monocytes toward to anti-inflammatory phenotype. The comprehensive understanding of the mechanism of action of GA may provide potential therapy target and useful insight for the development of new efficient drugs in the future.

\section{References}

1. Wingerchuk DM, Lucchinetti CF, Noseworthy JH (2001) Multiple sclerosis: current pathophysiological concepts. Lab Invest 81: 263-281.

2. Compston A, Coles A (2008) Multiple sclerosis. Lancet 372: 1502-1517.

3. Hafler DA, Slavik JM, Anderson DE, O'Connor KC, De Jager P, et al. (2005) Multiple sclerosis. Immunol Rev 204: 208-231.

4. Hohlfeld R, Wekerle H (2004) Autoimmune concepts of multiple sclerosis as a basis for selective immunotherapy: from pipe dreams to (therapeutic) pipelines. Proc Natl Acad Sci USA 101 Suppl 2: 14599-14606.

5. Kroenke MA, Carlson TJ, Andjelkovic AV, Segal BM (2008) IL-12- and IL23-modulated $T$ cells induce distinct types of EAE based on histology, CNS chemokine profile, and response to cytokine inhibition. J Exp Med 205: 15351541.

6. Matusevicius D, Kivisäkk P, He B, Kostulas N, Ozenci V, et al. (1999) Interleukin-17 mRNA expression in blood and CSF mononuclear cells is augmented in multiple sclerosis. Mult Scler 5: 101-104.

7. Uyttenhove C, Sommereyns C, Théate I, Michiels T, Van Snick J (2007) AntiIL-17A autovaccination prevents clinical and histological manifestations of experimental autoimmune encephalomyelitis. Ann N Y Acad Sci 1110: 330336.

8. Hofstetter HH, Ibrahim SM, Koczan D, Kruse N, Weishaupt A, et al. (2005) Therapeutic efficacy of IL-17 neutralization in murine experimental autoimmune encephalomyelitis. Cell Immunol 237: 123-130.

9. Fontenot JD, Gavin MA, Rudensky AY (2003) Foxp3 programs the development and function of CD4+CD25+ regulatory T cells. Nat Immunol 4: 330-336.

10. Haas J, Hug A, Viehover A, Fritzsching B, Falk CS, et al. (2005) Reduced suppressive effect of CD4+ CD25 high regulatory $T$ cells on the $T$ cell immune response against myelin oligodendrocyte glycoprotein in patients with multiple sclerosis. Eur J Immunol 35: 3343-3352.

11. Teitelbaum D, Webb C, Bree M, Meshorer A, Arnon R, et al. (1974) Suppression of experimental allergic encephalomyelitis in Rhesus monkeys by a synthetic basic copolymer. Clin Immunol Immunopathol 3: 256-262.

12. Arnon R, Sela M, Teitelbaum D (1996) New insights into the mechanism of action of copolymer 1 in experimental allergic encephalomyelitis and multiple sclerosis. J Neurol 243: S8-13.

13. Johnson KP, Brooks BR, Cohen JA, Ford CC, Goldstein J, et al. (1995) Copolymer 1 reduces relapse rate and improves disability in relapsing-remitting multiple sclerosis: results of a phase III multicenter, double-blind placebocontrolled trial. The Copolymer 1 Multiple Sclerosis Study Group. Neurology 45: 1268-1276.

14. Fridkis-Hareli M, Teitelbaum D, Gurevich E, Pecht I, Brautbar C, et al. (1994) Direct binding of myelin basic protein and synthetic copolymer 1 to class I major histocompatibility complex molecules on living antigen-presenting cells-specificity and promiscuity. Proc Natl Acad Sci USA 91: 4872-4876.

15. Fridkis-Hareli M, Strominger JL (1998) Promiscuous binding of synthetic copolymer 1 to purified HLA-DR molecules. J Immunol 160: 4386-4397.

16. Aharoni R, Teitelbaum D, Arnon R, Sela M (1999) Copolymer 1 acts against the immunodominant epitope $82-100$ of myelin basic protein by $\mathrm{T}$ cell receptor antagonism in addition to major histocompatibility complex blocking. Proc Natl Acad Sci USA 96: 634-639.

17. Murphy KM, Reiner SL (2002) The lineage decisions of helper T cells. Nat Rev Immunol 2: 933-944
18. Neuhaus O, Farina C, Yassouridis A, Wiendl H, Then Bergh F, et al. (2000) Multiple sclerosis: comparison of copolymer-1- reactive T cell lines from treated and untreated subjects reveals cytokine shift from Thelper 1 to T helper 2 cells. Proc Natl Acad Sci USA 97: 7452-7457.

19. Paul WE, Seder RA (1994) Lymphocyte responses and cytokines. Cell 76: 241 251

20. Mosmann TR, Sad S (1996) The expanding universe of T-cell subsets: Th1 Th2 and more. Immunol Today 17: 138-146.

21. Neuhaus O, Farina C, Wekerle H, Hohlfeld R (2001) Mechanisms of action of glatiramer acetate in multiple sclerosis. Neurology 56: 702-708.

22. Aharoni R, Teitelbaum D, Sela M, Arnon R (1998) Bystander suppression of experimental autoimmune encephalomyelitis by $T$ cell lines and clones of the Th2 type induced by copolymer 1. J Neuroimmunol 91: 135-146.

23. Aharoni R, Eilam R, Domev H, Labunskay G, Sela M, et al. (2005) The immunomodulator glatiramer acetate augments the expression of neurotrophic factors in brains of experimental autoimmune encephalomyelitis mice. Proc Natl Acad Sci USA 102: 19045-19050.

24. Chen M, Gran B, Costello K, Johnson K, Martin R, et al. (2001) Glatiramer acetate induces a Th2-biased response and crossreactivity with myelin basic protein in patients with MS. Mult Scler 7: 209-219.

25. Ziemssen T, Kumpfel T, Klinkert WE, Neuhaus O, Hohlfeld R (2002) Glatiramer acetate-specific T-helper 1- and 2-type cell lines produce BDNF: implications for multiple sclerosis therapy. Brain-derived neurotrophic factor. Brain 125: 2381-2391.

26. Kantengwa S, Weber MS, Juillard C, Benkhoucha M, Fellay B, et al. (2007) Inhibition of naive Th1 CD4+ T cells by glatiramer acetate in multiple sclerosis. J Neuroimmunol 185: 123-129.

27. Duda PW, Schmied MC, Cook SL, Krieger JI, Hafler DA (2000) Glatiramer acetate (Copaxone) induces degenerate, Th2-polarized immune responses in patients with multiple sclerosis. J Clin Invest 105: 967-976.

28. Chen C, Liu X, Wan B, Zhang JZ (2009) Regulatory properties of copolymer $\mathrm{I}$ in Th17 differentiation by altering STAT3 phosphorylation. J Immunol 183: 246-253.

29. Viglietta V, Baecher-Allan C, Weiner HL, Hafler DA (2004) Loss of functional suppression by $C D 4+C D 25+$ regulatory $T$ cells in patients with multiple sclerosis. J Exp Med 199: 971-979.

30. Hong J, Li N, Zhang X, Zheng B, Zhang JZ (2005) Induction of CD4+CD25+ regulatory $T$ cells by copolymer-I through activation of transcription factor Foxp3. Proc Natl Acad Sci USA 102: 6449-6454.

31. Vieira PL, Heystek HC, Wormmeester J, Wierenga EA, Kapsenberg ML (2003) Glatiramer acetate (copolymer-1, copaxone) promotes Th2 cell development and increased IL-10 production through modulation of dendritic cells. J Immuno 170: 4483-4488.

32. Hussien Y, Sanna A, Söderström M, Link H, Huang YM (2001) Glatiramer acetate and IFN-beta act on dendritic cells in multiple sclerosis. J Neuroimmunol 121: 102-110.

33. Kim HJ, Ifergan I, Antel JP, Seguin R, Duddy M, et al. (2004) Type 2 monocyte and microglia differentiation mediated by glatiramer acetate therapy in patients with multiple sclerosis. J Immunol 172: 7144-7153.

34. Weber MS, Starck M, Wagenpfeil S, Meinl E, Hohlfeld R, et al. (2004) Multiple sclerosis: glatiramer acetate inhibits monocyte reactivity in vitro and in vivo. Brain 127: 1370-1378. 